\title{
Using a New Routing Metric in Paths Discovery Process for MDSR
}

\author{
Saher Manaseer, Esra'a Alhenawi, Shatha Awawdeh
}

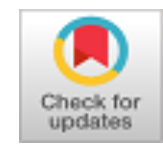

\begin{abstract}
Mobile Ad-hoc Network (MANET) is infrastructure-less network that consists of a set of mobile nodes. These nodes have limited power based on their batteries. Network lifetime is one of the most important challenges facing this type of networks; motivating many researchers to investigate alternatives that prolong the network lifetime. This paper proposes a new path selection metric that considers the ratio between the minimum residual energy of all route nodes and hop count value to select a rout in MDSR routing protocol. The discovered paths are checked periodically for ensuring their availabilities using special packets called DTC. Glomosim simulator is used to compare the modified MDSR protocol with the traditional MDSR and other existing protocols as well. Simulation results showed that the proposed routing protocol outperformed the traditional MDSR protocol in terms of network lifetime, packet delivery ratio and end to end delay. Moreover, it showed improved performance over other existing protocols in terms of packet delivery ratio and network lifetime.
\end{abstract}

Keywords: Manet, MDSR, Network, lifetime.

\section{INTRODUCTION}

Manet can be defined as a set of mobile nodes that can communicate with each other without using routers or specific infrastructure, each node in this network can work as a router for routing data from specific source to specific destination through one or more intermediate nodes. Routing process is done based on specific routing protocols. Routing protocol is a set of rules, aims at finding an efficient data transmission path across a network. There are various routing protocols that have been used in MANETs, and other that have been proposed for reaching to an efficient routing of data across the best paths in network based on multiple criteria's like number of hops, distance, capacity, load and path power.Routing protocols can be classified into two categories in MANETs: table based protocol (Proactive) and demand based protocols (Reactive) [1]. In table based protocol such as DSDV, WRP and GSR, each node maintains a routing table containing fresh lists of all possible routes to

Revised Manuscript Received on October 30, 2019.

* Correspondence Author

Dr. Saher Manaseer*, King Abdullah II school for Information Technology, Computer Science Department, The University of Jordan, Amman, 11942, Queen Rania Street, Jordan. (e-mail: saher@ju.edu.jo).

Esra'a Alhenawi, King Abdullah II school for Information Technology, Computer Science Department, The University of Jordan, Amman, 11942, Queen Rania Street, Jordan.

Shatha Awawdeh, King Abdullah II school for Information Technology, Computer Science Department, The University of Jordan, Amman, 11942, Queen Rania Street, Jordan

(C) The Authors. Published by Blue Eyes Intelligence Engineering and Sciences Publication (BEIESP). This is an open access article under the CC BY-NC-ND license (http://creativecommons.org/licenses/by-nc-nd/4.0/) all other nodes in the network by periodically distributing the routing tables through the network. On the other hand, in demand based protocol, routes to some destination must be discovered by a node only when it has a packet to send to that destination. AODV, DSR and SSR are examples of demand based protocols.In MANET, the overall network performance is affected by the power of batteries for the network nodes, which means that the management of battery power utilization in network nodes is very important factor to increase the network lifetime. Therefore, many researches in recent years proposed various power aware metrics in traditional routing protocols that depends on finding the shortest path with minimum hop count without taking into account the selected rout power, for decreasing power consumption and selecting paths that have maximum residual energy.This paper proposes a new metric to select the route path in MDSR routing protocol based on the ratio between the residual energy of the node and the hope counts then the proposed routing protocol is compared with the original MDSR, and other existing routing protocols as well to assess its efficiency.

\section{RELATED WORKS}

MANET can be defined as a set of mobile nodes that can communicate with each other without using routers or specific infrastructure, each node in this network can work as a router for routing data from specific source to a specific destination through one or more intermediate nodes. Routing process is done based on specific routing protocols.A routing protocol is a set of rules, aims at finding an efficient data transmission path across a network. There are various routing protocols that have been used in MANETs, and others that have been proposed for reaching to efficient routing of data across the best paths in network-based on multiple criteria's like number of hops, distance, capacity, load, and path power.Routing protocols can be classified into two categories in MANETs: table based protocol (Proactive) and demand based protocols (Reactive) [1]. In table-based protocol such as DSDV, WRP, and GSR, each node maintains a routing table containing fresh lists of all possible routes to all other nodes in the network by periodically distributing the routing tables through the network. On the other hand, in demand based protocol, routes to some destination must be discovered by a node only when it has a packet to send to that destination. AODV, DSR, and SSR are examples of demand based protocols.In MANET, the overall network performance is affected by the power of batteries for the network nodes, which means that the management of battery power utilization in network nodes is very important factor to increase the network lifetime. 
Therefore, many researches in recent years proposed various power aware metrics in traditional routing protocols that depends on finding the shortest path with minimum hop count without taking into account the selected rout power, for decreasing power consumption and selecting paths that have maximum residual energy. This paper proposes a new metric to select the route path in MDSR routing protocol based on the ratio between the residual energy of the node and the hope counts then the proposed routing protocol is compared with the original MDSR and other existing routing protocols as well to assess its efficiency.Many researchers have been done to update dynamic source routing (DSR) protocol in such a way to reduce the power consumption in one hand, and increase the network lifetime on the other hand. Some researches modified the way of route selection in DSR route discovery phase by taking the transmission power of the node and residual energy into account during the process of selecting routes between source and destination as proposed by Aye and Aung [1].Sharma [2] proposed a new routing protocol called Dynamic Source routing- power-aware routing DSR-PSR. The source node checks if a particular destination in the route cache exists, if the destination is found, then use that path to send a data packet, else route discovery process is used, in this process route request (PREQ) packet broadcasted by the source, each node adds to request node ID and battery status B-S. Battery status divided into three categories, based on these categories the number that must be added to the total battery status modified by 1 or 2 or 3 , after that the source node selects the path with high total battery status T-B-S, this protocol enhances the lifetime of the network and increases overall performance. Nagaraj et.al [3] proposed a new multipath routing protocol called (EM-DSR) which aimed at selecting multi-paths from source node to specific destination node based on maximum average residual energy which was equal to the ratio between the sum of the residual energy of all path nodes, and a number of nodes in that path. They used NS-2 Simulator to assess the proposed protocol performance by comparing it with the original single path DSR routing protocol, the results showed good improvement in network lifetime. Baisakh et.al [4] proposed routing protocol called Energy Conscious DSR (ECDSR). The route discovery phase in DSR was modified by discovering paths that have maximum energy rather than minimum hop count. The results showed that the proposed protocol outperformed the original DSR based on the number of dropped nodes and power consumption per packet delivery. Shankar et.al [5] proposed routing protocol as an enhancement to DSR routing protocol that called EDSR by minimizing the power consumption for nodes battery and prolong network lifetime.Reddy et.al [6] proposed an extension to their previous work which depends on delay and bandwidth in route selection decision by estimating the residual energy (based on receiving and transmitting the energy of nodes) and route stability called LET (based on velocity and node direction). They used NS-2 simulator where results showed better throughput and high efficiency. Bhadoria el at [7] presented an energy-aware model for wireless sensor network in which each sensor node randomly and alternatively stays in an active or sleep mode, there are two phases for active mode, the first one is full-active phase mode, which is used for data packet; transmits the sensed data packet, receive the packet and reply the received packet The second phase is semi-active which is only able to transmit/reply data, and there is a particular sensor node in a sleep mode that does not interact with any other node. The sensed node can switch from one phase to another randomly. In order to make the overloaded nodes sleep before a route link breaks Bella et al. in [8] proposed a new protocol depends on upgrade DSR protocol using dynamic path switching (DPS). The nodes send sleep request to the neighbor nodes for changing to sleep state for a while in two cases, the first case if it has forwarded packet for a long time and the second case if the battery level of the nodes arrives at the threshold value (which is between 20\%-30\%), the node cannot change state to sleep state unless received feedback from the neighbor, in this time the neighbor nodes try to find out an alternative route, if they find alternate route then send an acknowledgment for the source node to change state to sleep state and remove it from cache route table, but if the neighbor nodes cannot find alternate route then the source node still get a negative reply. Asleep scheduling protocol (SSP) was proposed by Anadamurugan and Venkatesh [9] to improve the lifetime of a dense sensor network by selecting a set of active nodes to be reset to sleep mode, the selection process is done by using a redundancy check algorithm, which is an algorithm to find out the neighbor nodes and check if these neighbors can reach to all other neighbors of the node, if the neighbor node cannot reach to all other neighbor nodes then reset this node to sleep mode for a period of time because of the node that are checked earlier will have a higher priority to go into sleep mode, after a period of time the sleeping nodes should wake up and receive a new reconfiguration information, this method is effective to increase the network lifetime. Recently, Jabri et.al [10] proposed new route selection criteria over MAODV routing protocol that depends on the ratio between minimum residual energy of all route nodes and hop count value. They checked the availability of selected paths periodically using DTC packets. In this paper, we will apply this criterion for routs discovery phase in MDSR routing protocol. The performance of an updated MDSR will be compared with the original MDSR, original MAODV and MEAODV that have been proposed in [10] in terms of end to end delay, packet delivery ratio and network lifetime using Glomosim simulator.

\section{MAIN PROCESSES IN THE MODIFIED MULTI-PATH DSR ROUTING PROTOCOL}

\section{A. Routs discovery process}

When one node needs to send a data packet, it initializes RREQ packets and sends it to all neighbor nodes using a flooding algorithm until it arrives to the destination node. The destination node receives the RREQ packets then initializes the RREP packet and sends it back to the source node, in this time the RREP packet saves the node ID and path power value which represents the minimum residual energy in the path. at the beginning, the path power equals the power of the first node in that path.

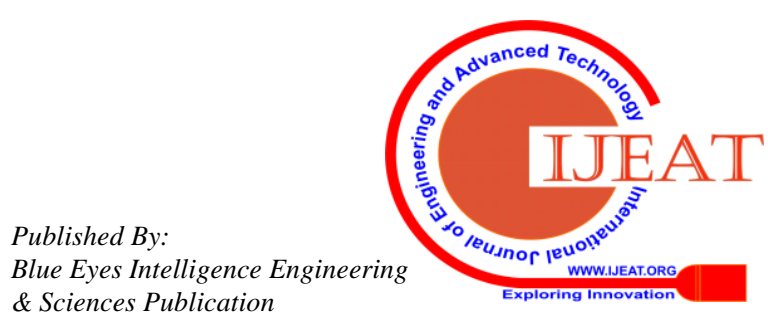


From the destination to the source node path, RREP packet checks the power of every node in the path if it is less than the path power or not, if the power of the node is less than the path power then the values of the node power are saved in place of the path power value, else the value of the path power remains the same.

When the RREP packets arrive to the source node, all discovered paths will be arranged in the route cache based on the ratio between path power (path power equal to the minimum residual energy of all path nodes) and hop count in decreasing order.

\section{B. Route maintenance process}

Source node sends data packets over the path with the highest ratio if it is still available. The availability of all discovered routes that have been stored in cache checked periodically for deleting routes that have been failed before using it. If failed occurs then the intermediate node sends ERRE packet that contains some information such as destination address and current path to the source node, then the source node deletes that path and searching in route cache for the alternate path for the same destination to send data over it if no alternative path is found, then re-apply the route discovery process

\section{IMPLEMENTATION PHASES FOR THE UPDATED DSR ROUTING PROTOCOL}

If you are using Word, use either the Microsoft Equation Editor or the MathType add-on (http://www.mathtype.com) for equations in your paper (Insert | Object | Create New | Microsoft Equation or MathType Equation). "Float over text" should not be selected.

There are five phases that have been accomplished to modifying the original single path DSR routing protocol.

Phase1: Implementing multi-path DSR routing protocol.

Phase2: Finding minimal nodal residual energy for each discovered route.

Phase3: Computing the ratio between minimal nodal residual energy and hop count.

Phase4: Arranging multi route in descending order based on the ratio (R) values from phase_3.

Phase5: Checking availability for each discovered route.

\section{A. Implementing multi-path DSR routing protocol}

Frequent link failures are common in MANET due to node's mobility and use of unreliable wireless channels for data transmission. Due to these factors, the multipath routing protocol becomes an important research issue. DSR is a reactive routing protocol. Multipath Dynamic Source Routing (MDSR) protocol is an enhancement part of the DSR protocol for computing multiple loop-free and link disjoint paths. To accomplish this phase, we modified the code of the single path DSR routing protocol in the Glomosim simulator by allowing it to take multiple paths in the route discovery phase. The main objective of this phase is to achieve efficient fault tolerance, i.e. quickly recovery from route failure. If one path fails, the protocol chooses an alternate route from other available paths that have been stored in the cache rather than sending new RREQ again (repeating route discovery).
In route maintenance phase, if an error occurs, we do not invoke a new route discovery whenever the selected path from the source to the destination fails as in single path-DSR routing protocol, instead we check if there exist another path from source to destination to use it directly.

\section{B. Finding minimal nodal residual energy for each discovered path}

Several changes are needed in the MDSR route discovery procedure to enable computation minimal nodal residual energy of each route between source-destination pairs. Therefore, the routing table must have a new field called Path Power. Each RREQ and RREP must have an additional field called power which represents the current node power and is calculated from the following equations (1) and (2) from [10]. Based on these equations results, the residual energy in each node in the route can be found, and the minimal node energy is considered to represent the energy of the path.

\section{Computing ratio between minimal nodal residual energy and hop count}

Ratio (R) for each discovered route must be calculated as a ratio between path power that had been calculated in phase 2 and hop count from source to destination through that route as shown in equation (3).

$$
\mathrm{R}=\text { Path Power / hop count }
$$

\section{Arranging multi route in descending order based on (R)}

The discovered routes will be ordered based on $\mathrm{R}$ value from phase 3 in descending order. If two or more routs have same $\mathrm{R}$ then these routes will be ordered randomly.

\section{E. Checking an availability for each discovered route}

At source node, each discovered path must be checked periodically if it still available to be used in failure cases for current rout by sending special packet called detected packet from source node to destination through all discovered paths, after the termination of specific time period, if this packet doesn't return to the source from destination through specific route, this route will be removed from discovered routes list. This process must be done regularly for ensuring availability of all routs that have been added in discovered routes list. Therefore, when current maintainable route fails, transmission will be directed to the best route (that has the highest $\mathrm{R}$ ) from the rest available paths.

\section{EXAMPLE ILLUSTRATES THE UPDATED MDSR}

This example illustrates how the updated MDSR routing protocol works:

Figure1 presents a network consists of 9 nodes where the source node (S) wants to send data to the destination node (D) using the updated MDSR routing protocol. This network has three paths between the source "S" and the destination "D" say P1, P2, P3 where P1 (2-3-4-D), P2 (6-7-D) and P3 (8-9-D). 


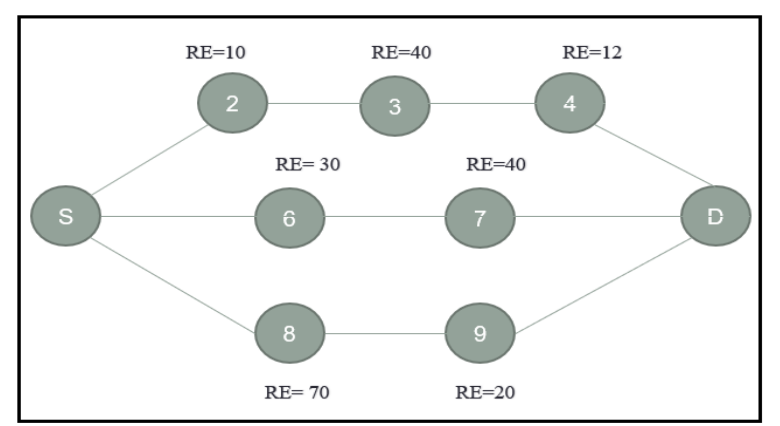

Example of Network using the updated MDSR protocol

\section{A. Route discovery process}

- Route discovery process begins when node "S" wants to send data to the destination node "D" but doesn't know any information about routes lead to that distention, therefore "S" sends RREQ packets to all of its neighbors $(2,6,8)$ asking for route to "D".

- Node D received the RREQ packet and sends back the reply packet RREP to the source node S over node 4, node 7 and node 9.

- For each path from D to S, RREP packet saves the node ID and compares the power of that node with path power in order to store the smallest value in path power. Initially the RREP packet saves the power of the first node in the path as the value of that path power (last value in RREQ).

- For the path D->9->8->S, path power calculated through the following steps:

Initially, D sends RREP packet to node 9 where path power = 20.

Node 9 forwards the RREP packet to node 8 (from which it receives RREQ), then compare the path power in RREP packet with residual power at node 8 which $=70$ Joule and save the smallest value in path power, so the path power still the same value which equal 20.

Now node 8 sends the RREP packet back to $S$ with path power $=20$ and hop count $=3$.

This process is done at the same time for all other paths

- The RREP packets arrive to the source node $\mathrm{S}$ which has the node ID and path power for that path.

- The source node $\mathrm{S}$ arrange the paths in route cache in decreasing order according to the ratio (R) between path power and hop count. In this example discovered paths arranged as shown bellow

P2: 6- $>7->D$ with path power $=30 / 3=10$

P3: $8->9->D$ with path power $=20 / 3=6.7$

P1: $2->3->4->$ D with path power $=10 / 4=2.5$

Periodically DTC packet sends over all discovered paths to check availability to these paths.

\section{B. Route maintenance process}

1. Send the data packet over the best path which has the largest $\mathrm{R}$ which is $\mathrm{P} 2$ in this example. P2: 6->7->D.

2. If the path failed, then the intermediate node sends RRER packet to the source node which delete the current path and check in its route cache for alternate path then take 8->9->D.

3. If the path failed, then the intermediate node sends RRER packet to the source node which delete the current path and check in its route cache for alternate path then take 2->3->4->D.

4. If the path failed, then delete the current path and check in route cache. If there is no rout to the node $\mathrm{D}$, then re-applies the rout discovery process.

\section{SIMULATION SETUPS}

This section provides the GloMoSim simulator setup that have been used to compare the performance of the updated MDSR (MEDSR), original MDSR, original MAODV and updated MAODV (MEAODV) proposed in [10].

Several experiments have been conducted to compare between different routing protocols in terms of end to end delay, packet ratio and network life time using the simulation setups that are shown in Table 1 with different number of nodes.

Table 1: Simulation Parameters

\begin{tabular}{|c|l|}
\hline \multicolumn{1}{|c|}{ Parameter } & \multicolumn{1}{|c|}{ Value } \\
\hline Simulation Area & $1000 \mathrm{~m} \times 1000 \mathrm{~m}$ \\
\hline Number of nodes & $10,30,50,150,250$ \\
\hline Routing protocol & $\begin{array}{l}\text { MEDSR, MDSR, MEAODV, } \\
\text { MAODV }\end{array}$ \\
\hline Traffic type & Constant Bit Rate (CBR) \\
\hline Number of packet & 150 \\
\hline Size of packet & $512 \mathrm{~b}$ \\
\hline
\end{tabular}

\section{EVALUATION AND RESULTS DISCUSSION}

Figure 2 below shows the results of seven experiments for various numbers of nodes small, medium and large numbers based on end to end delay for each protocol. Results show that MEDSR outperformed MDSR routing protocol while MEODV and MAODV routing protocols provides shorter delay than MEDSR due to nature of MDSR and MEDSR where in these protocols data have been stored as full path for any time in the cache while in MAODV only next hop stored; which leads to decrease end to end delay. As shown from this figure delay increased with corresponding to increasing in nodes number for all protocols. 


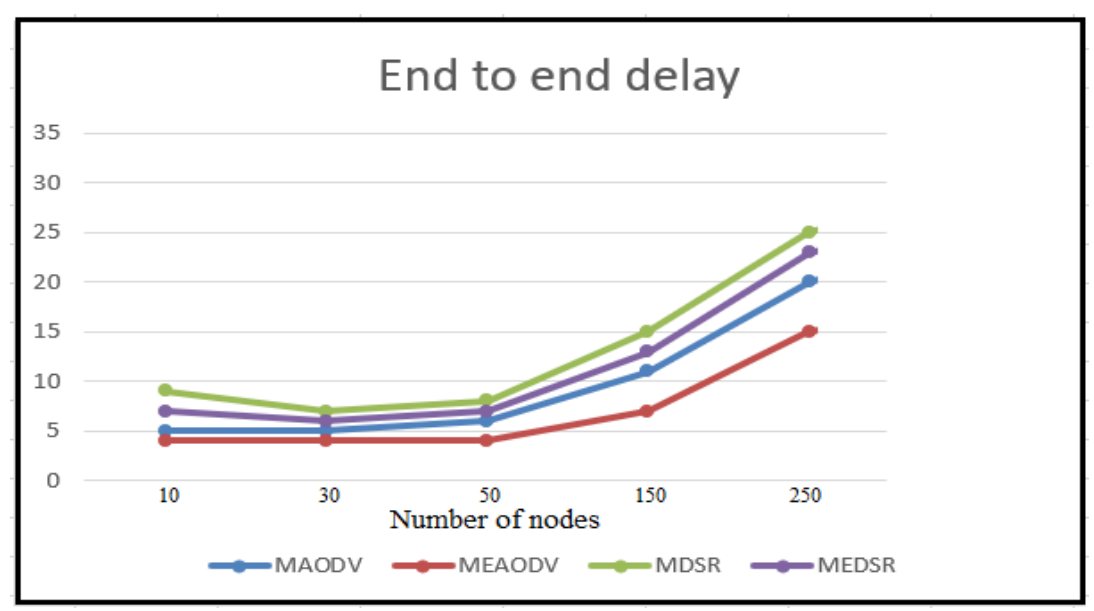

Fig. 2. end to end delay using different routing protocols

Figure3 below presents results based on packets delivery ratio for various number of nodes that represent three types of networks sparse, normal and dense networks where MEDSR outperformed all other protocols and satisfies value reached to $96 \%$ as number of nodes increased.

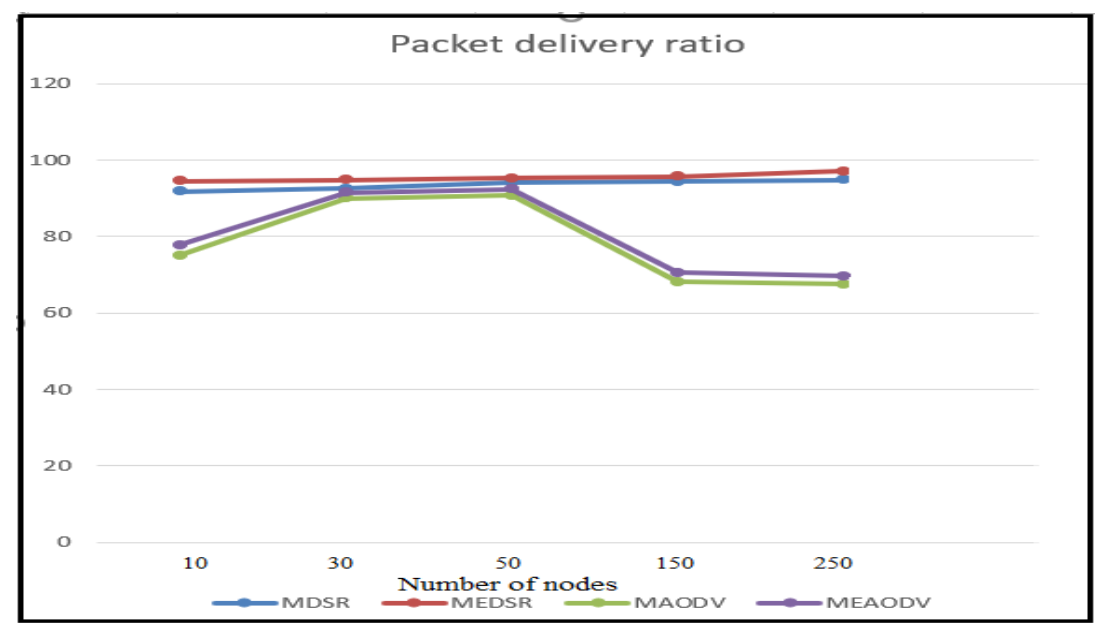

Fig. 3.packet delivery ratio using different routing protocols

Figure4 shows that MEDSR outperformed MDSR based on network lifetime with percentage equal 55.7\%, MEAODV with $52 \%$.

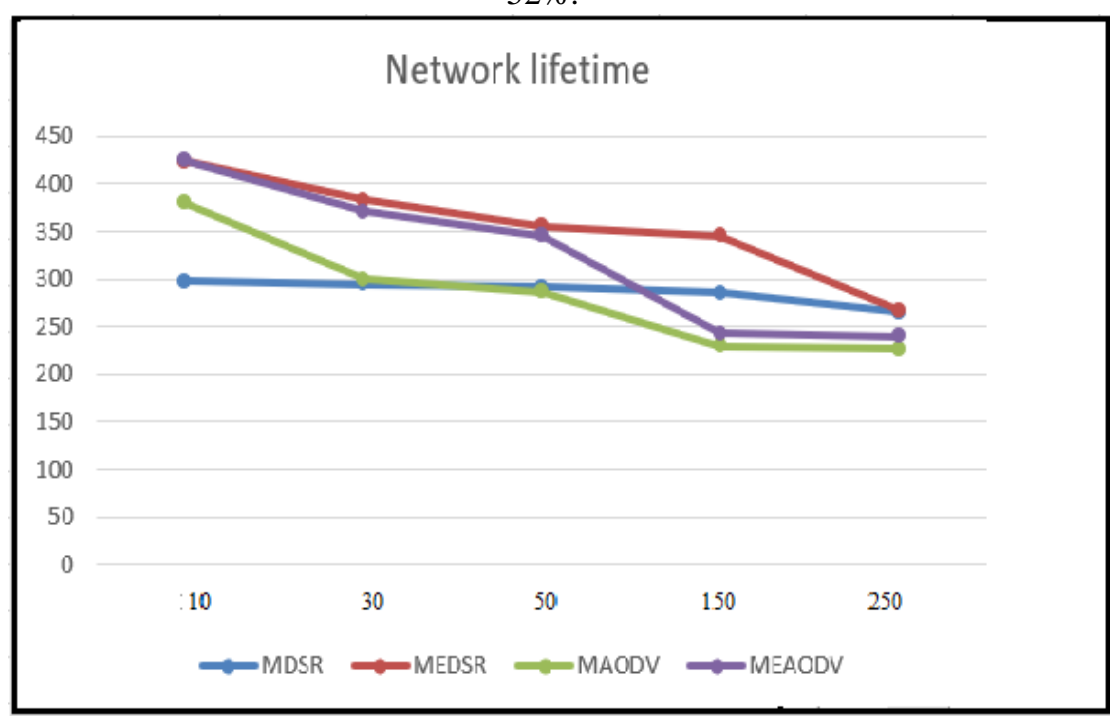

Fig. 4.Network lifetime using different routing protocols 


\section{CONCLUSION}

This paper applied an energy aware approach that has been proposed in [10] on MDSR routing protocol by selecting paths based on ratio between residual energy of node that has minimum residual energy in path from source to destination and hop count for that path. Glomosim simulator used for evaluating performance of an updated MDSR by comparing it with original MDSR, original MAODV and MEAODV routing protocol which proposed in [10]. Simulation results show that MEDSR outperformed all other protocols based on packet delivery ratio and network lifetime. MEDSR outperformed original MDSR based on end to end delay metric while MEAOD and original MAODV presents lesser delay than MEDSR.

\section{REFERENCES}

1. Malviya, L., Waoo, A. A. and Sharma, S. (2013) "New Load Balanced Multi-Path Dynamic Source Routing Protocol for Mobile Ad-HOC Network". International Journal of Computer Applications (0975-8887). DOI: 10.1109/ICCCNT.2013.6726571.

2. Sharma, K. and Sharma, V., (2014) "Energy Efficient Power Aware Multipath Dynamic Source Routing". IJCSNS International Journal of Computer Science and Network Security, 14(8), 47-52.

3. Nagaraj, m. Lutimath, Suresh and Naikodi, C., (2016) "Efficient Multipath Dynamic Source Routing Protocol for Mobile Ad Hoc Network', International Journal of Advanced Computational Engineering and Networking indexed in Eastern European Journal of Enterprise Technologies, ISSN: 2320-2106, 4(2016),10.

4. Baisakh N., R Patel, and Kumar, S., (2012) "Energy conscious DSR in MANET", 2nd IEEE International Conference on Parallel Distributed and Grid Computing (PDGC), Solan, 2012 page(s): 784 - 789, 6-8. DOI: 10.1109/PDGC.2012.6449922.

5. Shiva S., Varaprasad G., and Narayanagowda, S., (2013) "Implementing a new power aware routing algorithm based on existing dynamic source routing protocol for mobile ad hoc networks", published in IET Networks, 2013 vol. 3, Issue 2, pp. 137-142. DOI: 10.1049/iet-net.2013.0050.

6. Reddy, A. Pratapa, and N. Satyanarayana. (2017) "Energy-efficient stable multipath routing in MANET." Journal of Wireless Networks 23, no. 7 (2017): 2083-2091.

7. Robin Singh Bhadoria, R. S. B., Geetam Singh Tomar, G. S. T., and Sungmin Kang, S. K. (2014) "Proficient Energy Consumption Aware Model in Wireless Sensor Network". International Journal of Multimedia and Ubiquitous Engineering, 9(5), 27-36.

8. Bella, G., Costantino, G., Crowcroft, J. and Riccobene, S. (2013) "Enhancing DSR maintenance with power awareness". Computer Standards \& Interfaces, 35(1), 107-113.

9. Anandamurugan, S. and Venkatesh, C. (2009) "SSP: A sleep scheduling protocol for improving the lifetime of wireless sensor networks". In Control, Automation, Communication and Energy Conservation, INCACEC 2009. 2009 International Conference on (pp. 16). IEEE.

10. Jabri, R., Manaseer, S. and Alhenawi, E. M. (2018) "Using a New Routing Metric in Paths Discovery Process for MAODV". Open Access Library Journal 5, no. 04 (2018): 1

\section{AUTHORS PROFILE}

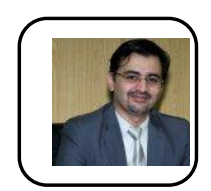

\section{Saher Manaseer}

Dr. Manaseer has a $\mathrm{PhD}$ in Computer Science from the Department of Computing Science at the University of Glasgow. His main area of research is Computer Networks and Embedded Systems. Currently, Dr. Manaseer is an active researcher in the field of Mobile Ad Hoc Networks. More specifically, his research on MANETs is focused on MAC layer protocols. Before obtaining his $\mathrm{PhD}$, He got his Masters in in Software Engineering from the University of Jordan.
Esra'a Alhenawi. Student at King Abdullah II school for Information Technology, Computer Science Department, The University of Jordan, Amman, 11942, Queen Rania. Street, Jordan

Shatha Awawdeh Student at King Abdullah II school for Information Technology, Computer Science Department, The University of Jordan, Amman, 11942, Queen Rania 